\section{Evaluar la escritura de ensayos argumentativos}

Claribel de Jesús Fernández Sunsín Colegio Notre Dame Nicaragua. clarafernandez.1984@gmail.com

\section{Evaluate the writing of argumentative essays}

(C) UNAN-Managua

Recibido: agosto 2017

Aprobado: octubre 2017

La autoevaluación y coevaluación son instrumentos de evaluación empleados en un proyecto didáctico que se aplicó con los estudiantes de undécimo grado. Aquí, se exponen los resultados de su puesta en práctica en la producción textual. Ciertamente, los estudiantes aprendieron a autoevaluarse y a coevaluar la escritura a través de la escucha atenta, el reconocimiento de sus aciertos y errores, el diálogo entre iguales con el propósito de corregir los equívocos y mejorar sus textos.

Los estudiantes que participaron en este proceso didáctico aprendieron a argumentar por escrito, reconocieron que son artífices de su propio aprendizaje y que la escritura es una forma de permanecer en la memoria de todos los que lean sus ensayos argumentativos. En sus escritos se observó la temática de la naturaleza humanana, el valor de la persona y el contexto social en que están inmersos. También, aplicaron ejercicios metacognitivos para autorregular su proceso de escritura.

La secuencia didáctica titulada "Escribamos ensayos convincentes: un proyecto de escritura", se aplicó a estudiantes de undécimo grado de educación secundaria, quienes presentaban dificultades en la estructuración de la información y en la redacción de ensayos. El trabajo por proyectos promueve la interacción, la capacidad de modificar el texto con apoyo del docente. En esta línea Camps (1996) sostiene: "La interacción promueve la capacidad de gestión del texto, que es primero compartida y de acuerdo con la tesis Vigotskiana, podrá llegar a ser interiorizada y autónoma" (p.67). La perspectiva anterior explica el carácter interactivo de los proyectos de escritura. Las habilidades (lectura, escritura, habla y escucha) se aprenden durante el proceso, se interiorizan hasta llegar a aplicarse de forma independiente por el escritor del texto.

La intervención didáctica consistió en la aplicación del proceso de composición del ensayo argumentativo. Se explicó a los estudiantes los conceptos, características, importancia y estructura de la tipología objeto de estudio. Se les invitó a utilizar las diferentes estrategias, herramientas de evaluación del proyecto didáctico y a aplicar el proceso de escritura de forma recursiva. De igual manera, los alumnos reconocieron el uso reflexivo de la lengua, observaron, analizaron el escrito (metalenguaje) y apreciaron la funcionalidad de los saberes aprendidos.

Además, se consideró la evaluación como una fase integral que valora la habilidad de expresión escrita del autor. Esta se apoya en los principios de la evaluación: tiene en cuenta diferentes edades de desarrollo de la capacidad de escritura, el estímulo que se le dio, la finalidad y el tipo de texto usado (Serafini, 1995). Es decir, que al evaluar el escrito el docente considera la capacidad del discente que ha redactado el texto. 


\section{Revista de Lengua y Literatura. Vol.4, núm. 1, enero-junio 2018}

La evaluación de los aprendizajes fue auténtica porque se aplicaron los conocimientos adquiridos en el contexto comunicativo en el que los estudiantes se encontraban. En este sentido, López, V. et.al. (2011) exponen que "suele entenderse como evaluación auténtica el hecho de que las técnicas, instrumentos y actividades de evaluación estén claramente aplicadas en situaciones, actividades y contenidos reales del aprendizaje que se busca" (p. 32).

Es decir, que las herramientas de evaluación se utilizaron en la práctica real con el propósito que los estudiantes autoevaluaran sus escritos y coevaluaran los borradores de sus compañeros de clases, a través de pautas y rúbricas de evaluación para el proceso (borradores de ensayo) y para el producto (ensayo final).

Primero, se aplicó a los estudiantes la evaluación diagnóstica, después la formativa y sumativa a los estudiantes. En relación con la primera, los alumnos produjeron su escrito diagnóstico, el cual consideraron como ensayo argumentativo. Esta evidencia se analizó para valorar si era necesario un proceso didáctico para la mejora de la competencia escrita. Efectivamente, fue necesaria su aplicación, porque a través de esta se trabajó la evaluación formativa que permitió a los estudiantes regular y mejorar su proceso de escritura mediante el uso de herramientas de autoevaluación y coevaluación. Finalmente, se aplicó la evaluación sumativa con el uso de la rúbrica del ensayo argumentativo final y el escrito producido por los estudiantes al concluir el proceso de escritura.

La coevaluación favorece el trabajo cooperativo, porque los estudiantes interactúan entre sí. Según Fernández, A. (2006) "permite desarrollar competencias académicas, profesionales, habilidades interpersonales, de comunicación y modificar actitudes" (p. 46). En definitiva, ambas formas de evaluación contribuyen al desarrollo integral de los estudiantes; cabe señalar que al docente le compete participar en la validación de los instrumentos de evaluación con el colectivo de docentes de la misma área, para garantizar su fiabilidad.

Las estrategias de evaluación aplicadas procuraron mejoras cualitativas en los aprendizajes de los estudiantes. Esto se logró a través de procesos ricos en los que cada alumno aplicó a su escrito herramientas de evaluación objetivas. El rol del profesor consiste en facilitar estos procesos de autoevaluación y coevaluación. El primero favorece el aprendizaje autónomo, continuo y proporciona a los estudiantes un mayor grado de control sobre su aprendizaje. Y el segundo desarrolla la habilidad de comunicación y actitudes de escucha y cooperación.

Asimismo, la información que aportan la autoevaluación y coevaluación sobre el proceso de enseñanza-aprendizaje de los estudiantes en relación con la competencia escrita, facilita el análisis de los motivos que provocan los avances o dificultades de los alumnos en el aprendizaje. Por otro lado, sirve para que el estudiante tome conciencia de las estrategias que le resultan más adecuadas para producir su texto. En consecuencia, conduce a buscar medidas de apoyo apropiadas para que los alumnos mejoren la calidad de sus escritos; a la vez permite revisar y regular los distintos factores que interactúan en el proceso de aprendizaje: las características del alumno, la especificidad de la tarea y la metodología empleada.

Ahora bien, la forma de evaluación de los centros educativos no se desliga del sistema de evaluación, desde el punto de vista de la influencia que el último ejerce sobre aquella. En este sentido, el docente prepara el contexto que favorezca un sistema activo de evaluación. Lo hace a través del diálogo con los estudiantes. En efecto, la conversación entre alumnos y alumno-maestro ha de generar confianza, actitud reflexiva y crítica, elementos esenciales para la autoevaluación, coevaluación y retroalimentación del docente en el proceso de enseñanza de escritura. 


\section{Claribel de Jesús Fernández}

Planificar la escritura... pp. 26-35

Además, las herramientas anteriores enriquecen el aprendizaje de los estudiantes.

Evidentemente, los instrumentos de evaluación (Pautas de autoevaluación, coevaluación, rúbricas y listas de cotejo) pretenden investigar el nivel de logro cualitativo-cuantitativo según el interés del evaluador. Fernández, M. (2005) resalta la ventaja de la evaluación cualitativa como instrumento individualizado (para uso de cada docente en su aula), porque su importancia radica en que suministra información la cual permite construir planes de ayuda al alumno. Considerando esta perspectiva, la aplicación de los instrumentos permite al docente observar logros y dificultades de los estudiantes en el proceso de enseñanza-aprendizaje de la escritura y ayuda a preparar una intervención didáctica para la mejora de la competencia escrita de los estudiantes.

De igual manera, se enseñó a los estudiantes a autorregular su aprendizaje, se utilizó el portafolio de escritura, el cual les permitió autorregular su proceso y reflexionar sobre las estrategias aplicadas para la expresión escrita. Cabe señalar que el portafolio es una recopilación y selección de evidencias que demuestran un proceso de aprendizaje y el desarrollo de unas determinadas capacidades (López, 2011). Para esto el docente explicó a los alumnos el uso más amplio del instrumento, de tal modo que recopilara evidencias de las dificultades, las mejoras en la escritura del ensayo argumentativo y ejercicios de metacognición, como muestra de la reflexión realizada por los estudiantes sobre cómo estaban aprendiendo y qué estrategias les estaban facilitando la redacción de sus ensayos.

Además, el portafolio constituyó una alternativa para recopilar información: mostró las habilidades y logros de los estudiantes: cómo piensan, producen o crean sus textos. Es decir, permitió identificar los aprendizajes de conceptos, procedimientos y actitudes relacionadas con la escritura. El portafolio implicó desarrollo, variedad y se utilizó como forma de evaluación, coevaluación y autoevaluación (Villalobos, 2002). Es decir, que la aplicación de este instrumento permitió monitorear la evaluación del proceso de aprendizaje de los estudiantes, de tal manera que se realizaron cambios y reflexiones del propio aprendizaje en relación con la redacción de ensayos con adecuada estructuración de la información.

También, se aplicó la metacognición durante el proceso didáctico, esta implica un conjunto de procesos mentales que se utilizan para guiar una tarea o una actividad. Se trata de analizar la mejor forma de hacer la tarea. La hemos definido como pensar sobre el propio pensamiento para dirigirlo y ayudarnos a razonar mejor. En este sentido, es una acción autorreflexiva: consiste en pensar cómo estamos trabajando (Sota, J. et.al. 2006). Es decir, que el alumno se autointerroga antes, durante y después de la tarea; con el propósito de establecer un sistema de autorregulación del proceso de pensamiento. Por ejemplo: ¿Cómo debo proceder? ¿Lo estoy haciendo bien? ¿Qué necesito hacer si no entiendo algo? ¿Con qué objetivo estoy leyendo este material? ¿En qué orden debo trabajar? ¿Cuánto tiempo tengo para completar la tarea?

Naturalmente, el estudiante analiza su trabajo y se autointerroga, porque la metacognición le lleva a hacer una revisión de lo que ha producido durante una tarea específica. Por ejemplo: ¿Qué ha pensado, recordado e imaginado? En este sentido, refleja qué procesos mentales se han puesto en práctica para la escritura del ensayo. Gracias a la reflexión, el alumno se hace más consciente de lo que aprendió (el producto), cómo aprendió (el proceso) y para qué aprendió (la finalidad del aprendizaje). En efecto, los estudiantes se responsabilizaron de su propio aprendizaje. 
Los estudiantes aplicaron la autorregulación, la cual hace referencia al uso, guía y control de la cognición. Ahora bien, ¿Cuáles son los recursos cognitivos de un estudiante? Son su capacidad de centrar su atención en la tarea y sostener esa atención durante un tiempo prolongado (20-30 minutos) la capacidad de llevar a cabo dos o más tareas a la vez eficientemente, de recordar bien lo que se ha escuchado en clases y aprovechar el tiempo apropiadamente. A continuación, se muestran ejemplos de ejercicios realizados por los estudiantes. Con la aplicación de estos, los estudiantes fortalecieron su capacidad de reflexión, el análisis, autocrítica y actitud propositiva para hacer las enmiendas en su ensayo.

Los elementos de la lista son ejercicios metacognitivos para el portafolio del alumno.

- La revisión del texto me ha servido para...

- He tenido dificultades para...

- Cómo he superado las dificultades...

- He vuelto a reescribir mi texto porque...

- En relación a la escritura he aprendido que...

En consecuencia, la autorregulación permitió a los estudiantes modificar las acciones durante el proceso de escritura, planificar el escrito, monitorear el trabajo, revisar, corregir, evaluar los resultados y las estrategias que utilizaron para la producción textual y aplicar lo aprendido cuando tengan tareas de producción escrita.

En el siguiente ejemplo de ejercicio metacognitivo se consideró el estado de ánimo del estudiante frente a las estrategias aplicadas, su actitud en relación al proceso de escritura que estaba viviendo, el análisis de los medios que le ayudaron a escribir su ensayo y reconocimiento de la función que tiene la argumentación para aplicarla en su contexto comunicativo. Con el uso de la metacognición, los estudiantes desarrollaron la capacidad de autorregulación de su proceso de aprendizaje, reflexión de sus actitudes y reconocimiento de las estrategias empleadas para la escritura del ensayo.

La siguiente lista es un ejercicio metacognitivo para el portafolio del alumno.

- ¿Cómo me sentí con la aplicación del portafolio?

- Mi actitud durante el proceso de escritura ha sido...

- Lo que me ha ayudado a escribir este texto ha sido...

- La argumentación escrita me sirve para...

- Lo que aprendí del proceso de escritura del ensayo argumentativo es...

- El uso del portafolio me ha servido para...

Es importante señalar que la aplicación de los ejercicios de metacognición estimuló la reflexión sobre la utilidad de los aprendizajes en su vida académica, permitió a los sujetos del aprendizaje reconocer si se habían apropiado de conocimientos, habilidades y actitudes relacionadas con la escritura, retomar e integrar ideas que no consideraron durante la elaboración del bosquejo, retomar los objetivos del escrito y monitorear su proceso de escritura. El ejercicio se aplicó durante la producción textual, esto permitió a los estudiantes la autorregulación de su aprendizaje. Obsérvese los siguientes ejemplos: 
Planificar la escritura del ensayo argumentativo me ha servido para...

- La función que cumple mi ensayo argumentativo es...

- Revisar el bosquejo es importante porque...

- Es conveniente que la próxima vez tenga en cuenta...

El ensayo se evaluó durante el proceso de forma dinámica y pertinente, en lugar de un escrito pasivo como antes se había hecho. En perspectiva de Cassany (1994) se utiliza una evaluación más cualitativa, basada en técnicas de observación y recogida de información. Se hacen entrevistas a los alumnos, se les observa mientras escriben, se analizan comparativamente los borradores que producen (p.297). En concreto, la información recolectada a través de la observación (instrumento de investigación) y la conversación con los estudiantes, permitió reconocer los avances, los procesos que realizaron y sus actitudes en relación con la escritura. Con los primeros borradores producidos por los estudiantes se aplicó una evaluación formativa del ensayo argumentativo.

Para mejorar la escritura del ensayo fue apropiado considerar que el proceso de redacción se encarga de transformar el proyecto de texto, que hasta ahora era solo un esquema semántico, una representación jerárquica en un discurso lineal e inteligible (Cassany et.al. 1994). Considerando el aporte del autor, los escritores planificaron, elaboraron borradores, revisaron, reelaboraron fragmentos del ensayo y estructuraron la información de forma ordenada: introducción (presentación de la tesis) desarrollo (argumentos y contraargumentos) y conclusión (reiteración de la tesis e invitación a la acción). En efecto, la aplicación del proceso de escritura de forma recursiva garantizó mejoras en la organización de la información.

Además, se trabajó con errores de escritura detectados en los escritos diagnósticos. Para superarlos no fue suficiente con citar el tipo de error cometido y el método para corregirlo. Los alumnos necesitaban ver la forma apropiada o una posible versión rectificada de lo que se ha hecho de forma errada, para comprender cómo deben hacerlo (Cassany, 1995). Por tal razón, la docente proporcionó comentarios sobre el escrito regularmente. También, se explicó al estudiante el error, la forma de corregirlo y observar las rectificaciones que el docente opera en su texto, para que él las pueda realizar de forma autónoma.

A continuación se describen las fases trabajadas por los estudiantes.

Tanto en la fase planificación como en la textualización del ensayo los estudiantes hicieron uso del bosquejo, lo revisaron con apoyo de la pauta de coevaluación y autoevaluación, se observó que leían, conversaban, borraban y reescribían su texto. Dialogaron en parejas sobre sus bosquejos, la interacción entre ellos favoreció el desarrollo de las habilidades de lectura, escritura, habla y escucha atenta. De igual forma, consultaron su material de estudio para aclarar dudas. Desde el inicio conocieron la pauta de evaluación del ensayo final, lo que les permitió avanzar gradualmente para producir un ensayo argumentativo coherente y bien estructurado.

Para la corrección del escrito observaron las correcciones realizadas por la docente y sugerencias para mejorar la estructuración de la información en el ensayo. Los instrumentos de evaluación contribuyeron a la mejora de los escritos de los estudiantes, porque se hicieron responsables de su propio aprendizaje y asimilaron el uso de las rúbricas y pautas de evaluación. En suma, los alumnos se benefician más de la evaluación periódica que cuando esperan un examen final. 
La pauta de autoevaluación del primer borrador del ensayo argumentativo permitió a los estudiantes reconocer si habían utilizado el bosquejo de la planificación para escribir su borrador de ensayo. Si la tesis era coherente, si los argumentos del esquema los habían utilizado y si consideraron la organización de la información (tesis, argumentos y conclusión). Se tomaron su tiempo para revisar sus borradores, se aseguraron de comprender bien cuáles eran sus errores y cómo corregirlos. Sus borradores tenían errores de coherencia: falta de relación entre las ideas, ausencia de conclusión, falta de relación entre los argumentos y la tesis. También, recibieron retroalimentación del docente. Obsérvese el ejemplo de los criterios de la pauta evaluativa del primer borrador del ensayo argumentativo aplicada por los estudiantes.

Tabla 1.

Pauta para autoevaluar el primer borrador del ensayo argumentativo.

\begin{tabular}{|l|l|l|l|l|}
\hline & Criterios & SI & NO & A medias \\
\hline \multirow{2}{*}{ Tesis } & $\begin{array}{l}\text { 1. He hecho un borrador siguiendo la } \\
\text { planificación pero introduciendo } \\
\text { algún cambio. }\end{array}$ & & & \\
\hline Argumentos & $\begin{array}{l}\text { 2. Escribí la tesis de forma clara y } \\
\text { completa. }\end{array}$ & & & \\
\hline & 3. La tesis es coherente. & & \\
\hline & $\begin{array}{l}\text { 4. La tesis aparece al inicio del escrito. } \\
\text { 5. Utilicé todos los argumentos de la } \\
\text { lista de planificación }\end{array}$ & & & \\
\hline & $\begin{array}{l}\text { 6. Hay argumentos suficientes para } \\
\text { desarrollar la tesis. }\end{array}$ & & & \\
\hline $\begin{array}{l}\text { 7. Escribí argumentos basados en } \\
\text { opiniones de algún científico o } \\
\text { personalidad entendida en el tema. }\end{array}$ & & & \\
\hline $\begin{array}{l}\text { 8. Redacté argumentos justificados con } \\
\text { datos objetivos o ejemplos. }\end{array}$ & & & \\
\hline $\begin{array}{l}\text { 9. Hay argumentos en cada párrafo con } \\
\text { ejemplos o explicaciones. }\end{array}$ & & & \\
\hline $\begin{array}{l}\text { 10. Utilicé conectores entre las ideas del } \\
\text { párrafo. }\end{array}$ & & & \\
\hline 11. La conclusión reitera la tesis. & & \\
\hline $\begin{array}{l}\text { 12. La conclusión hace reflexionar al } \\
\text { lector. }\end{array}$ & & & \\
\hline Conclusiones & & & \\
\hline
\end{tabular}


La aplicación de la secuencia didáctica permitió registrar errores y mejoras de los estudiantes en relación con la estructuración de la información. Hubo mejora en los ensayos de los discentes, ellos lo afirman en los ejercicios metacognitivos, en los cuales hacen una reflexión sobre los conocimientos adquiridos, los procedimientos aplicados, su actitud de cara al proceso de escritura, las dificultades presentadas y cómo las superaron durante la aplicación del proyecto. Nótese la perspectiva de una de las estudiantes:

La revisión del texto me ha servido para corregir mis errores, mejorar lo que escribí, analizar mejor mi objetivo para que mi ensayo sea coherente. En relación a la escritura he aprendido el uso de los conectores, mis argumentos tienen un poco más de coherencia, espero mejorar mucho más.

Fue gratificante conocer las opiniones de los estudiantes respecto a la utilidad del proyecto didáctico. Sobre todo fue satisfactorio observar que ellos reconocieron sus errores, los aspectos a corregir en el escrito y cómo estaban realizando las mejoras. De igual manera, se generó una actitud positiva frente al proceso de escritura, que facilitó su aplicación. En este sentido, reflexionaron sobre su aprendizaje y las estrategias que les permitieron estructurar su ensayo. Principalmente, se logró observar que los estudiantes analizaron la funcionalidad de la argumentación escrita, lo que aprendieron del proceso de escritura del ensayo argumentativo y la utilidad del portafolio. Léase la opinión de uno de los estudiantes:

La argumentación escrita me sirve para expresar opiniones, informar sobre temas importantes y generar una reflexión. Lo que aprendí del proceso de escritura del ensayo argumentativo es: escribir ensayos coherentes, con vocabulario adecuado. El portafolio me sirvió de guía para escribir el ensayo, usar conectores y la normativa APA para citar bibliografía.

Durante la escritura del texto los estudiantes evidenciaron que estaban aprendiendo a estructurar la información, porque emplearon el portafolio de escritura, utilizaron el bosquejo para redactar borradores del ensayo y el material de estudio de la argumentación, los conectores y el ensayo. Asimismo, aplicaron pautas de autoevaluación, coevaluación y rúbrica del proceso de escritura, para revisar y corregir su ensayo argumentativo. Cabe destacar que se fortaleció la revisión y retroalimentación del docente respecto al escrito para la mejora continua del mismo. A continuación aparece un ejemplo de criterios de la pauta de coevaluación del segundo borrador de ensayo argumentativo: 
Revista de Lengua y Literatura. Vol.4, núm. 1, enero-junio 2018

Tabla 2.

Pauta de coevaluación del segundo borrador del ensayo argumentativo

\begin{tabular}{|l|l|l|l|}
\hline Elementos de la argumentación & SI & NO & A medias \\
\hline 1. ¿Queda claro el receptor del texto? & & & \\
\hline 2. ¿Queda claro a quién se escribe el texto? & & & \\
\hline 3. ¿Definí claramente mi afirmación? & & & \\
\hline 4. ¿Acepté opiniones de los oponentes? & & & \\
\hline Estructuración de la información & & & \\
\hline Tesis & & & \\
\hline 1. La tesis tiene coherencia. & & & \\
\hline 2. La tesis es clara y directa. & & & \\
\hline Argumentos & & & \\
\hline 1. Hay argumentos suficientes para desarrollar la tesis. & & & \\
\hline 2. Hay argumentos coherentes en cada párrafo. & & & \\
\hline $\begin{array}{l}\text { 3. Hay argumentos basados en opiniones de algún } \\
\text { científico o personalidad entendida en el tema. }\end{array}$ & & & \\
\hline $\begin{array}{l}\text { 4. Hay argumentos justificados con datos objetivos o } \\
\text { ejemplos. }\end{array}$ & & & \\
\hline 5. Hay conectores que marcan el orden de los argumentos & & & \\
\hline $\begin{array}{l}\text { 6. Se contrastan los argumentos con los contraargumentos } \\
\text { en el mismo párrafo }\end{array}$ & & & \\
\hline Conclusión & & & \\
\hline 1. La conclusión reitera lo planteado en la tesis. & & \\
\hline 2. La conclusión conduce al lector a una reflexión. & & \\
\hline
\end{tabular}

\section{Plan para la corrección}

\section{Discutimos antes de reescribir el texto}

1. ¿Qué apartados o párrafos cambiarías o volverías a escribir?

2. ¿Qué añadirías al texto?

3. ¿Qué suprimirías? ¿Por qué? 
En conclusión, la aplicación de los instrumentos de autoevaluación y coevaluación permitió que los estudiantes tomaran conciencia del trabajo que realizaron, porque reflexionaron sobre su propio aprendizaje a través de la aplicación de los ejercicios metacognitivos durante la producción de su ensayo. Los elementos anteriores fueron relevantes para que los estudiantes mejoraran la estructuración de la información en el ensayo. Además, al comparar los escritos diagnósticos con los ensayos producidos durante el proyecto didáctico hubo mejora, porque se observó que la mayoría produjo la tesis con coherencia, argumentos suficientes para desarrollarla, la conclusión del texto y finalmente la presentación de la antología de ensayos.

Además, la interacción entre los estudiantes generada por la coevaluación favoreció el desarrollo de las macrohabilidades de lectura comprensiva, escritura, oralidad y escucha de las opiniones de los demás. De igual forma, los escritores lograron potenciar las siguientes habilidades: aprender haciendo a través de la metacognición, identificar errores para la mejora del escrito, reconocer tipos de argumentos en textos modelo, determinar argumentos que enfatizan la tesis, llegar al lector meta, analizar la utilidad del proceso de escritura y uso del portafolio.

Por las razones antes planteadas, es preciso reconocer que es provechoso formar estudiantes con capacidad de autoevaluar sus propios escritos, compartir en equipo sus perspectivas para la mejora del trabajo. Coevaluar la escritura y asumir responsabilidades sobre su proceso de aprendizaje. No es una utopía, los cambios se generan desde las aulas de clases con el fin de formar usuarios competentes de la lengua. Por su puesto, que implica más trabajo para el colectivo de docentes de una determinada asignatura académica. Sin embargo, es preciso mejorar las prácticas pedagógicas de forma continua, compartir de forma propositiva con el claustro de docentes las estrategias que fueron funcionales, con el sincero propósito de mejorar lo que hasta ahora se ha hecho para evaluar la escritura. 


\section{$\longrightarrow$ REFERENCIAS -}

Camps, A. (1996) Proyectos de lengua entre la teoría y la práctica. Cultura y educación 2,4357. Madrid: Aprendizaje.

Cassany, D. et.al. (1994). Enseñar lengua. Barcelona: GRAO

Cassany, D. (1995). Reparar la escritura. Barcelona: GRAO

Fernández, M. (2005). Evaluación y cambio educativo: el fracaso escolar. Madrid: Morata.

Fernández, A. (2006). Metodologías activas para la formación de competencias. Revista Educatio Siglo XXI (24) 35-56

López, V. (2011). Evaluación formativa y compartida en Educación superior. España: Narcea S.A.

Villalobos, J. (2002 enero-marzo). Portafolios y reflexión: instrumentos de evaluación en una clase de escritura. Revista Educere. 5(16) 390-396

Serafini, M. (1995). Cómo redactar un tema. Didáctica de la escritura. Barcelona: Paidós. Sota, J., et. al. (2006). Guía de estrategias metacognitivas para desarrollar la comprensión Lectora. Perú: Firmat. SAC. 